\title{
Review
}

Pathobiology

\section{International Telepathology: Promises and Pitfalls}

\author{
Navid Farahani ${ }^{a} \quad$ Michael Riben ${ }^{b} \quad$ Andrew J. Evans ${ }^{c} \quad$ Liron Pantanowitz $^{a}$ \\ ${ }^{a}$ Department of Pathology, University of Pittsburgh School of Medicine, Pittsburgh, Pa., and ${ }^{b}$ Department of \\ Pathology and Laboratory Medicine, The University of Texas MD Anderson Cancer Center, Houston, Tex., USA; \\ 'Department of Pathology, Laboratory Medicine Program, University Health Network, Toronto General Hospital, \\ Toronto, Ont., Canada
}

\section{Key Words}

Digital pathology $\cdot$ Informatics $\cdot$ International

telepathology $\cdot$ Whole-slide imaging

\begin{abstract}
Innovative technologies for digital imaging and telecommunications are changing the way we deliver health care. Telepathology collaborations are one example of how delivering remote pathology services to patients can benefit from leveraging this change. Over the years, several academic and commercial teleconsultation networks have been established. Herein, we review the landscape of these international telepathology efforts and highlight key supportive factors and potential barriers to successful cross-border collaborations. Important features of successful international telepathology programs include efficient workflows, dedicated information technology staff, continuous maintenance, financial incentives, ensuring that all involved stakeholders are satisfied, and value-added clinical benefit to patient care. Factors that plague such telepathology operations include legal/regulatory issues, sustainability, and cultural and environmental issues. Pathologists, vendors and laboratory accreditation agencies will need to embrace and capitalize on this new paradigm of international telepathology accordingly.

(c) 2016 S. Karger AG, Basel
\end{abstract}

\section{KARGER}

E-Mail karger@karger.com www.karger.com/pat

\section{Introduction}

Significant technological gains continue to fuel the process of globalization, with direct effects on the health care industry [1]. For example, it is often easier to move images across geographic borders and across continents than it is to send biological material (e.g. tissue slides and/ or blocks) or transport pathologists or patients. Thus, telepathology, which facilitates access to pathology experts around the world, continues to demonstrate considerable growth. Telepathology is of great benefit to underserved and rural areas where there is a significant shortage of pathologists. In these areas, not only is there a high demand for diagnostic consultation, but also a need for continuing education and guidance on patient management. Telemedicine in Africa has proven to be a very useful conduit of health care [2]. A review of telepathology consultation between the University of Pittsburgh Medicine (UPMC) in the USA and KingMed Laboratories in China revealed that in many cases patient management was significantly impacted as a result of expert diagnoses [3]. In recent years, international teleconsultation has grown and provided a novel source of revenue.

Over the years, there has been a plethora of international telepathology ventures. Table 1 lists several of these collaborations [4]. One of the earliest of these undertakings was iPATH, developed by the University of Basel in 
Table 1. Examples of international telepathology solutions, services and collaborations

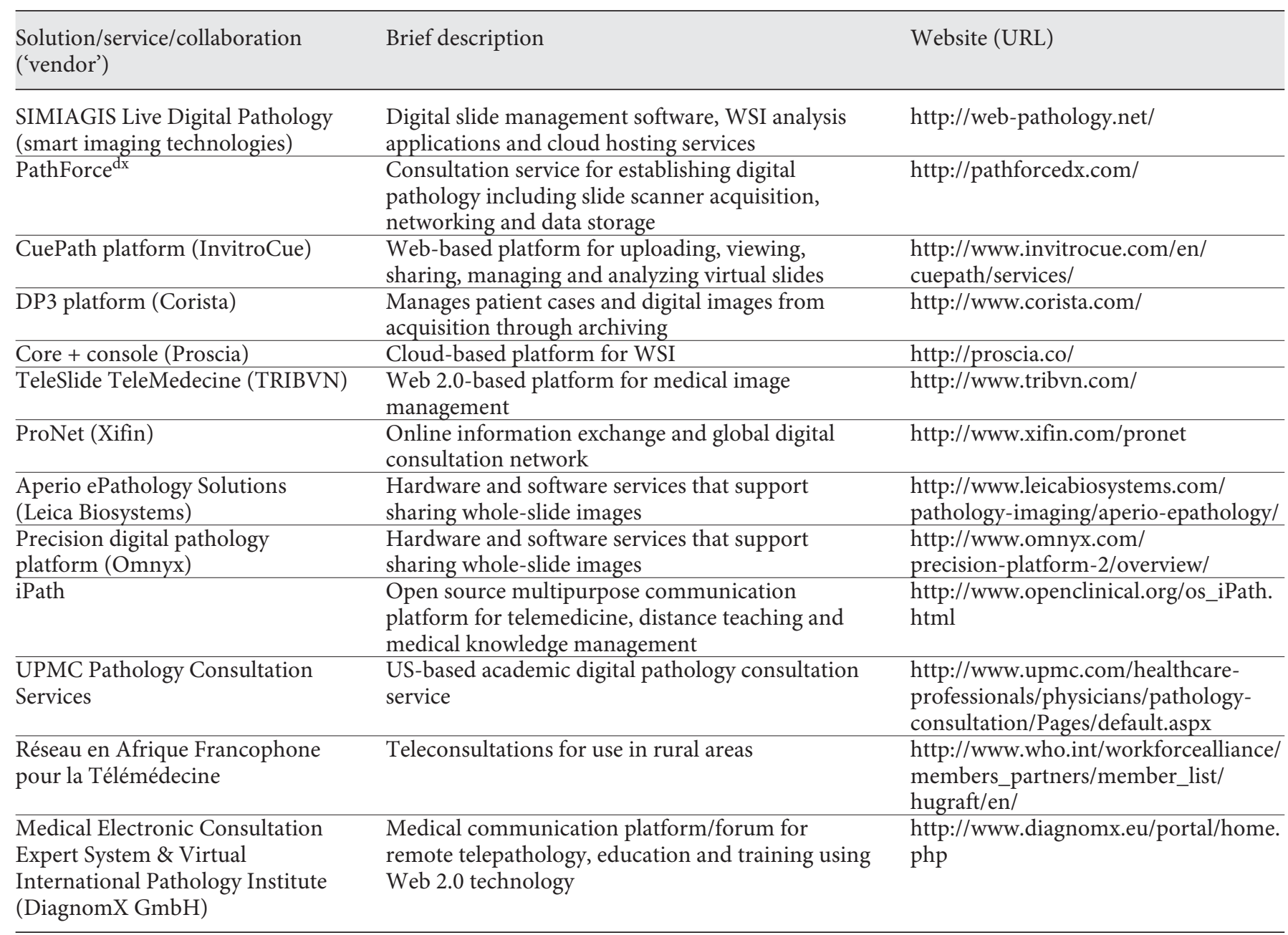

$2001[5,6]$. iPATH was built using open source software to service over 150 user groups around the world. This global network has enabled the examination of over 15,000 telepathology cases. MECES (the Medical Electronic Consultation Expert System), a similar international telepathology platform, exploits Web 2.0 and whole-slide imaging (WSI) technologies [7]. VIPI (the Virtual International Pathology Institute), which utilizes MECES for both data input and output, is an international, electronically based institute of pathology, which provides expert consultation, 'liable' diagnoses, and numerous additional features like automated access to reference libraries, automated measurement of images and automated language translation $[1,8]$. More recently, several international telepathology networks have been established by large academic institutions (e.g. the University
Health Network in Canada, UPMC, MD Anderson in Texas and the Massachusetts General Hospital in Boston). Commercial vendors have also begun offering collaborative telepathology portables, cloud services and established business partnerships with several pathology groups (e.g. PathCentral, AccelPath, Aperio ePathAccess, Corista and XIFIN).

Technologies utilized to practice international telepathology have evolved since their inception in the late 1980s [9]. Initial efforts relied on store-and-forward systems where static images were the mainstay of image exchange. By 2001, the transplant pathology service at UPMC had accumulated experience with teleconsultation for over 3,000 static images. Digital consultation for these cases demonstrated acceptable diagnostic concordance between digital and glass diagnoses [10]. More 
contemporary telepathology platforms have adopted WSI. WSI enables remote viewing in one of two ways: digital files can either be accessed on a remotely shared server owned by the host facility (or third party), or transmitted and uploaded (e.g. via a web portal) to a server that is owned by the consultant group $[10,11]$. The former arrangement requires strong cooperation between medical, administrative and information technology (IT) divisions on both ends, in addition to permission to access foreign servers. The latter arrangement may result in time delays due to image transmission; however, image viewing is less likely to suffer from network delays or firewall issues. Newer platforms to support telepathology have begun using diagnostic viewers, cloud services, more open access platforms, plug-in technology and even mobile cellular devices.

Evaluation of international telemedicine collaborations in general has revealed several factors deemed vital to success [12]. These include low operating costs, utilization of simple technologies, bidirectional communication, incentive-based programs, locally responsive services, strong team leadership, appropriate training and user acceptance. Barriers to international telemedicine include legal/regulatory issues (e.g. ensuring that all parties adhere to applicable laws and international safe harbor regulations), sustainability factors (e.g. costs, inconsistent use and poor scalability), cultural factors (e.g. language, trust and limited resources) and environmental factors (e.g. IT infrastructure, network limitations and time zones). It is anticipated that many of the current obstacles will likely be addressed as technology advances and standardization of international contracts become more commonplace [13]. Eventually, geographic borders will hopefully no longer serve as a barrier to the remote delivery of pathology services.

\section{Technology}

Over the last two decades, there has been a significant shift in the types of technologies deployed in the practice of telepathology. The Weinstein Telepathology System Classification describes 12 distinct classes of telepathology systems [14]. For simplicity, these modes can be divided into static (store-and-forward), dynamic (aka realtime) or hybrid systems that combine elements of both static and dynamic imaging.

Early international telepathology efforts relied heavily on static (snapshot) imaging platforms. These systems captured, stored and forwarded galleries of digital images

International Telepathology: Promises and Pitfalls for remote diagnosis. In order to be effective, such platforms had to link digital images with metadata such as patient identifiers, and, if necessary, relevant clinical, laboratory and radiological information. For static image telepathology, the individual who selects representative microscopic fields at the sending site must possess basic knowledge in order to determine which regions of interest need to be imaged. The major advantages of static image telepathology include relatively low start-up costs and minimal equipment maintenance. Moreover, image files are small and hence often easier to manage and store. These are all-important factors when dealing with resource-poor countries. However, there are numerous disadvantages (e.g. sampling errors, limited fields of view and focus problems) to this method that likely account for its low diagnostic accuracy when compared with dynamic telepathology $[15,16]$.

Dynamic telepathology platforms enable pathologists to view entire glass slides by remotely controlling motorized microscope functions, including slide navigation (in the $\mathrm{x}$ - and $\mathrm{y}$-axes) and focusing (along the $\mathrm{z}$-axis). By the 1990s, this particular method of robotic telepathology was very popular [17-19]. However, these dynamic telepathology platforms were more expensive, required the use of proprietary hardware/software by both sending and receiving sites, and hence lacked interoperability. Robotic telepathology systems have been infrequently used to share images across international borders. In addition to the disadvantages alluded to above, their use for realtime telemicroscopy is often impractical when dealing with time zones and in countries with limited telecommunication infrastructure. With robotic microscopy capability recently being incorporated into whole-slide scanners, it is likely that this form of telepathology may be better exploited in the near future.

WSI has been used not only for international telepathology, but also heavily employed to foster educational and research collaborations among international parties. WSI systems produce large, high-resolution digital images of entire glass slides that can be stored on web-accessible servers or in the cloud to be viewed over the Internet. WSI permits access to an entire glass slide or a set of slides at various magnifications [20,21]. Contemporary WSI scanners can potentially capture a $1.5 \times 1.5 \mathrm{~cm}$ tissue section at $\times 20$ magnification in under $1 \mathrm{~min}$. For international telepathology, this facilitates the scanning of large volumes of slides. Certain WSI scanners offer z-scanning, which permits slides to be digitized with multiple focal planes. Such z-stacked digital slides are better suited for interpreting cytology teleconsultations. Viewing whole- 
slide images is usually faster than using dynamic telepathology, especially when using a powerful computer and high-speed network connection [22]. The primary disadvantages of WSI telepathology include the expensive costs of the equipment, image archiving demands for large digital files and image viewer compatibility for different file formats. OpenSlide provides an interface to read virtual slides in many (e.g. .svs, .svslide, .tif, .bif, .ndpi and .scn) but not all formats [23].

\section{Collaboration Benefits}

Telepathology, international and domestic efforts alike, facilitate remote access to specialist expertise, which in turn results in better and often more efficient patient care [19-22]. Successful telepathology practice is directly related to advances in computing technology and the Internet (e.g. web 2.0 tools). One of the primary strengths offered by telepathology is the potential to improve operational efficiency. It is often easier to move an image than it is to move a pathologist or patient. A recent study by Ho et al. [24] projected millions of dollars in cost saving for their health care system that could be realized from the adoption of digital pathology due to improved productivity (e.g. consolidated histology operations and workload balancing) and more accurate diagnoses (e.g. facilitation of teleconsultation). Telepathology eliminates the costly, inefficient and time-consuming process of having to manually transport delicate glass slides via third-party courier services. For certain countries (e.g. China), human tissues may not be allowed to cross international borders, making telepathology the only means of obtaining a second opinion. For institutions that lack inhouse specialty expertise, teleconsultation is critical [25]. In these instances, telepathology capabilities may be deemed invaluable, especially by patients, who can reap the benefits of remote expert consultation [2]. Telepathology collaborations have proven to be beneficial for developing countries because such partnerships have also offered a mechanism for sharing of best practices for laboratories, providing education and creating the opportunity for research. In some cases, broad institutional telemedicine efforts have combined telepathology with other services, such as teledermatology. For pathologists, travel to remote locations to provide care can be avoided. By being able to work anywhere and at any time, pathologists' experience enhanced job satisfaction, and they feel less isolated when working in remote regions and retain access to educational activities [22].

\section{Keys to Success}

Successful international telepathology depends on several factors. The key stakeholders include pathologists, as well as administrative and IT personnel. Pathologists, the primary users of telepathology systems, are arguably the most critical stakeholders. Hence, any telepathology platform under consideration must be reliable, intuitive and flexible enough to meet their needs. One concern is that digital pathology disrupts their existing workflow. Telepathology platforms should not only be easy to use, but pathologists will also need to be well trained to use them. This is especially true for those professionals who are reluctant to switch to digital pathology or may be technophobic. For administrative stakeholders, it is important that telepathology improves patient care while concurrently improving operations and return on investment. IT buy-in and support is critical to successful telepathology practice to ensure that storage needs, system compatibility, network capabilities and security are not overlooked. Despite lowered costs and recent advances in digital storage infrastructure, large-scale use of WSI can easily deplete server capacity. Several vendors now offer cloud-hosting services. However, there are still concerns related to security and cloud computing. Threats related to cloud security include reports of prior corporate data breaches, data loss, account or service traffic hijacking, public-facing application programming interfaces and shared technology.

In recent years, major steps have been undertaken to improve and encourage the adoption of telepathology by the pathology community. In 1999, the American Telemedicine Association put forth telepathology guidelines, which were subsequently updated in 2014 [26]. Additional telepathology guidelines using WSI were provided by the College of American Pathologists, Royal College of Pathologists in Britain and the Canadian Association of Pathologists [27-30]. These guidelines not only address the varying technologies available and different clinical applications, but also discuss important issues related to maintenance, quality assurance, security and regulations.

\section{Barriers}

Apart from financial and technology barriers (e.g. electricity supply and reliable telecommunication bandwidth in rural or underserved areas), there are also regulatory and cultural factors that may impede international telepathology [31]. There is some degree of uncertainty 
regarding the liability of health professionals when delivering care across borders [32]. While there are no specific provisions set forth in the European Union legislation, in the United States physician licenses are not portable. There is a draft bill currently before the US Congress to facilitate telemedicine endeavors by addressing such legal barriers [33-35]. Apart from these regulatory concerns, uncertainty regarding medicolegal implications poses another barrier. The adoption of explicit and/or implied consent should be included during telepathology contract negotiations. This can be especially challenging in countries with high rates of illiteracy and limited familiarity with the technologies used to share information across borders [36]. Additional legal issues associated with telepathology stem from data security concerns regarding the confidentiality of medical information on the Internet [37-40]. Potential solutions for confidentiality include the anonymization of data through a variety of mechanisms (i.e. removal of patient identifiers or limiting user access to stored patient information). Platforms that support secure data transfer and data encryption are an essential element of successful telepathology programs $[41,42]$.

Other important factors to delivering quality care across borders include local technical support, reliable connectivity, good image quality, quality assurance measures, monitoring timeliness of reporting, database maintenance and the availability of bidirectional communication between parties. Many international laboratories with limited resources encounter difficulties in the production of optimal, high-quality glass slides that have well-stained tissue. Consequently, telepathology consults may be limited due to these poor quality slides. Furthermore, poorly prepared tissues and slides (e.g. tissue present outside the coverslip or tissue folds) may affect optimal scanning and thereby hamper interpretation [3]. Another limiting factor is when experts do not have additional data about the case, such as pertinent clinical information, radiology images or measurements, and the results of ancillary studies (e.g. flow cytometry or molecular information). Lack of these metadata can make it hard for an expert consultant to provide a comprehensive opinion for challenging cases. Sustainability of crossborder telepathology services has been a major problem for several collaborations. Historically, these failed telepathology programs have been small-scale collaborations initiated by individuals or poorly funded nongovernmental organizations. The implementation of international telepathology undertakings can be established using simple, low-cost technologies, with low initiation and main- tenance costs. Expensive setups, like satellite-based services, are now being replaced by cheaper and more ubiquitous alternatives (e.g. the Internet and cellular devices). For international telepathology, there may also be language and cultural barriers. Therefore, it is important to have a pathologist champion and IT expert on both sides that are familiar with local/regional issues. They need to be able to easily communicate and foster collaboration and trust, and deal with troubleshooting when problems arise. Obtaining patient and public trust also plays an essential role in long-term international telepathology collaborations.

\section{Conclusion}

International telepathology efforts have primarily been driven by the need to improve access to specialist pathology services in low- and middle-income countries where there is a shortage of pathologists. To date, telepathology collaborations have mainly offered teleconsultation services (i.e. providing second opinions on challenging cases) and education. Fruitful collaborations highlight the importance of satisfying the needs of all key stakeholders, providing financial incentives and adequate training, employing sustainable and flexible telepathology platforms, adopting WSI and fostering trust between groups. Factors that hinder international telepathology are numerous and include finances, lack of local IT support, legal and regulatory issues, data security and confidentiality concerns, as well as language barriers. Nevertheless, it is anticipated that the practice of international telepathology will continue to grow as more vendors begin to offer practical solutions to support these collaborations, and studies begin to show their positive impact on global patient care.

References

1 World Health Organization: Trade, Foreign Policy, Diplomacy and Health: Globalization. Geneva, World Health Organization, http:// www.who.int/trade/glossary/story043/en/ (accessed April 5, 2015)

2 Wamala DS, Augustine K: A meta-analysis of telemedicine success in Africa. J Pathol Inform 2013;4:6.

- 3 Zhao C, Wu T, Ding X, Parwani AV, Chen H, Lauro GR, Feng X, Wu S, Yousem S, Pantanowitz L: International telepathology consultation: two years of experience between the University of Pittsburgh Medical Center (UPMC) and KingMed (China). J Pathol Inform 2014;5:S33-S34. 
4 Park S, Parwani AV, Aller RD, Banach L, Becich MJ, Borkenfeld S, Carter AB, et al: The history of pathology informatics: a global perspective. J Pathol Inform 2013;4:7.

-5 Oberholzer M, Christen H, Haroske G, Helfrich $\mathrm{M}$, Oberli $\mathrm{H}$, Jundt $\mathrm{G}$, et al: Modern telepathology: a distributed system with open standards. Curr Probl Dermatol 2003;32: 102-114.

6 Brauchli K, Kurt B, Martin O: The iPath telemedicine platform. J Telemed Telecare 2005; 11:3-7.

7 Kayser K, Borkenfeld S, Djenouni A, Kayser G: History and structures of telecommunication in pathology, focusing on open access platforms. Diagn Pathol 2011;6:110.

8 Virtual International Pathology Institute (VIPI): DiagnomX GmbH. http://www.diagnomx.eu/vipi/home.php (accessed September 29, 2015).

-9 Pantanowitz L, Wiley CA, Demetris A, Lesniak A, Ahmed I, Cable W, et al: Experience with multimodality telepathology at the University of Pittsburgh Medical Center. J Pathol Inform 2012;3:45.

10 Minervini MI, Yagi Y, Marino IR, Lawson A, Nalesnik M, Randhawa P, et al: Development and experience with an integrated system for transplantation telepathology. Hum Pathol 2001;32:1334-1343.

11 Romero Lauro G, Cable W, Lesniak A, Tseytlin E, McHugh J, Parwani A, Pantanowitz L: Digital pathology consultations - a new era in digital imaging, challenges and practical applications. J Digit Imaging 2013;26:668-677.

-12 Saliba V, Legido-Quigley H, Hallik R, Aaviksoo A, Car J, McKee M: Telemedicine across borders: a systematic review of factors that hinder or support implementation. Int J Med Inform 2012;81:793-809.

13 Carter AB: Stepping across borders into the future of telepathology. J Pathol Inform 2011; $2: 24$.

14 Weinstein RS, Descour MR, Chen L, Bhattacharyya AK, Graham AR, Davis JR, et al: Telepathology overview: from concept to implementation. Hum Pathol 2001;32:1283-1299.

15 Kayser K, Szymas J, Weinstein R: Telepathology and Telemedicine: Communication, Electronic Education, and Publication in eHealth. Berlin, VSV Interdisciplinary Medical Publishing, 2005.

-16 Dunn BE, Choi H, Recla DL, Kerr SE, Wagenman BL: Robotic surgical telepathology between the Iron Mountain and Milwaukee Department of Veterans Affairs Medical Centers: a 12-year experience. Hum Pathol 2009; 40:1092-1099.

17 Halliday BE, Bhattacharyya AK, Graham AR, Davis JR, Leavitt SA, Nagle RB, et al: Diagnostic accuracy of an international static-imaging telepathology consultation service. Hum Pathol 1997;28:17-21.

18 Nordrum I, Engum B, Rinde E, Finseth A, Ericsson $\mathrm{H}$, Kearney M, et al: Remote frozen section service: a telepathology project in northern Norway. Hum Pathol 1991;22:514-518.

19 Kaplan KJ, Burgess JR, Sandberg GD, Myers CP, Bigott TR, Greenspan RB: Use of robotic telepathology for frozen-section diagnosis: a retrospective trial of a telepathology system for intraoperative consultation. Mod Pathol 2002;15:1197-1204.

20 Wilbur DC, Madi K, Colvin RB, Duncan LM, Faquin WC, Ferry JA, et al: Whole-slide imaging digital pathology as a platform for teleconsultation: a pilot study using paired subspecialist correlations. Arch Pathol Lab Med 2009;133:1949-1953.

21 Furness P: A randomized controlled trial of the diagnostic accuracy of internet-based telepathology compared with conventional microscopy. Histopathology 2007;50:266-273.

-22 Evans AJ, Runjan C, Clarke BA, Sidney C, Ghazarian DM, Tim-Rasmus K, et al: Primary frozen section diagnosis by robotic microscopy and virtual slide telepathology: the University Health Network experience. Hum Pathol 2009;40:1070-1081.

23 Goode A, Gilbert B, Harkes J, Jukic D, Satyanarayanan M: OpenSlide: a vendor-neutral software foundation for digital pathology. J Pathol Inform 2013;4:27.

24 Ho J, Ahlers SM, Stratman C, Aridor O, Pantanowitz L, Fine JL, et al: Can digital pathology result in cost savings? A financial projection for digital pathology implementation at a large integrated health care organization. J Pathol Inform 2014;5:33

25 Weinstein RS, Graham AR, Richter LC, Barker GP, Krupinski EA, Lopez AM, et al: Overview of telepathology, virtual microscopy, and whole slide imaging: prospects for the future. Hum Pathol 2009;40:1057-1069.

26 Pantanowitz L, Dickinson K, Evans AJ, Hassell LA, Henricks WH, Lennerz JK, et al: American Telemedicine Association clinical guidelines for telepathology. J Pathol Inform 2014;5:39.

27 Pantanowitz L, Sinard JH, Henricks WH, Fatheree LA, Carter AB, Contis L, et al: Validating whole slide imaging for diagnostic purposes in pathology: guideline from the College of American Pathologists Pathology and Laboratory Quality Center. Arch Pathol Lab Med 2013;137:1710-1722.

28 Thrall MJ, Wimmer JL, Schwartz MR: Validation of multiple whole slide imaging scanners based on the guideline from the College of American Pathologists Pathology and Laboratory Quality Center. Arch Pathol Lab Med 2015;139:656-664.
29 Lowe J: Telepathology: Guideline from the Royal College of Pathologists. http://www.rcpath.org/Resources/RCPath/Migrated $\% 20$ Resources/Documents/G/G026_Telepathology_Oct13.pdf (accessed April 10, 2015).

30 Canadian Association of Pathologists Telepathology Guidelines Committee, Bernard C, Chandrakanth SA, Cornell IS, Dalton J, Evans A, et al: Guidelines from the Canadian Association of Pathologists for establishing a telepathology service for anatomic pathology using whole-slide imaging. J Pathol Inform 2014;5:15.

31 Bagayoko CO, Müller H, Geissbuhler A: Assessment of Internet-based tele-medicine in Africa (the RAFT project). Comput Med Imaging Graph 2006;30:407-416.

-32 Ahmed SN, Mann C, Siddiqui F, Sheerani M, Syed NA, Snyder T, Enam SA, Boling W: Experiences from an international tele-epilepsy collaboration. Can J Neurol Sci 2009;36:582586.

33 Fitzgerald R: Medical regulation in the telemedicine era. Lancet 2008;372:1795-1796.

34 LeRouge C, Garfield MJ: Crossing the telemedicine chasm: have the U.S. barriers to widespread adoption of telemedicine been significantly reduced? Int J Environ Res Public Health 2013;10:6472-6484.

35 Mosquera M: Sen. Udall drafting bill to kill telemedicine barriers. Government Health IT, 2012. http://www.govhealthit.com/news/ udall-drafting-bill-remove-telemedicinebarrier (accessed February 2, 2012).

- 36 Nakashima N, Shimizu S, Okamura K, Hahm JS, Kim YW, Han HS, et al: Development of a broadband telemedical network based on internet protocol in the Asia-Pacific region. Methods Inf Med 2007;46:709-715.

37 Martínez Álvarez M, Chanda R, Smith RD: How is telemedicine perceived? A qualitative study of perspectives from the UK and India. Global Health 2011;7:17.

38 Williams BH, Mullick FG, Butler DR, Herring RF, O'leary TJ: Clinical evaluation of an international static image-based telepathology service. Hum Pathol 2001;32:1309-1317.

-39 Zbar RI, Otake LR, Miller MJ, Persing JA, Dingman DL: Web-based medicine as a means to establish centers of surgical excellence in the developing world. Plast Reconstr Surg 2001;108:460-465.

40 Zolfo M, Arnould L, Huyst V, Lynen L: Telemedicine for HIV/AIDS care in low resource settings. Stud Health Technol Inform 2005; 114:18-22.

41 Jarvis L, Stanberry B: Teleradiology: threat or opportunity? Clin Radiol 2005;60:840-845.

42 Swinfen R, Swinfen P: Low-cost telemedicine in the developing world. J Telemed Telecare 2002;8(suppl 3):63-65. 